Check for updates

Cite this: RSC Adv., 2018, 8, 9141

Received 4th January 2018 Accepted 14th February 2018

DOI: $10.1039 / \mathrm{c} 8 \mathrm{ra00074c}$

rsc.li/rsc-advances

\section{A urinary metabolomics (GC-MS) strategy to evaluate the antidepressant-like effect of chlorogenic acid in adrenocorticotropic hormone- treated rats $\uparrow$}

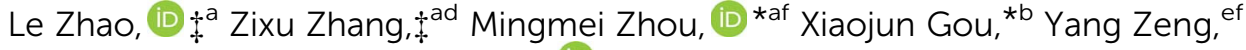 \\ Jing Song, ${ }^{a}$ Weini $\mathrm{Ma}^{\mathrm{a}}$ and Ying Xu (D)
}

Major depressive disorder (MDD) is a chronic recurring illness that seriously affects human health. Chlorogenic acid (CGA), an important polyphenol extracted from Eucommia ulmoides Oliver bark, has been reported to have anti-depression, neuroprotection, memory improvement and other pharmacological effects. However, little is known about the underlying mechanisms of CGA on the treatment of depression. Here, we investigated the antidepressant-like effects of CGA on an adrenocorticotropic hormone $(\mathrm{ACTH})$-treated rat model. Thirty-two male Wistar rats were randomly divided into four groups: normal diet group (N), ACTH-treated model group (M), memantine positive control group $(M+M e m)$ and CGA intervened group ( $M+C G A)$. Sucrose preference tests (SPTs) and open-field tests (OFTs) were performed to evaluate depressive-like behaviors. Memantine (30 $\mathrm{mg} \mathrm{kg}^{-1}$ ) and CGA (500 $\mathrm{mg} \mathrm{kg}^{-1}$ ) administration dramatically increased hedonic behaviors of the rats in SPT. The scores of crossing and rearing were significantly increased in the $M+$ Mem group and $M+$ CGA group. These results of the behaviour tests might be suggestive of antidepressant-like effects. Moreover, memantine and CGA reversed the levels of serum 5-hydroxytryptamine $(5-H T), A C T H$, corticotropinreleasing hormone $(\mathrm{CRH})$, and dopamine $(\mathrm{DA})$ that were altered in $\mathrm{ACTH}$-treated rats. Based on a GC-MS metabolomic approach, significant differences in the metabolic profile were observed in ACTH-treated rats compared with the control group, as well as the $M+C G A$ group and $M+$ Mem group compared with the ACTH-treated group. A total of 19 metabolites were identified for the discrimination of normal rats and ACTH-treated rats, and 12 out of 19 differential metabolites were reversed with CGA intervention. Combined with pattern recognition and bioinformatics, nine perturbed metabolic pathways, including energy metabolism, neurotransmitter metabolism, and amino acid metabolism, were identified based on these metabolites. These integrative studies might give a holistic insight into the pathophysiological mechanism of the ACTH-treated depressive rat model, and also showed that CGA has antidepressant-like activities in ACTH-treated rats, providing an important drug candidate for the prevention and treatment of tricyclic anti-depressant treatment-resistant depression.

\section{Introduction}

Depression is a severe psychiatric and mental illness with a rapid growth rate. Up to $30-40 \%$ of depressive patients do not respond to treatment with the existing antidepressants, ${ }^{\mathbf{1}}$ and people undergoing antidepressant treatment may confront a delayed onset of therapeutic effects, high recurrence, and side effects. ${ }^{2,3}$ Therefore the demand space for new
${ }^{a}$ Center for Chinese Medicine Therapy and Systems Biology, Shanghai University of Traditional Chinese Medicine, 1200 Cailun Road, Pudong District, Shanghai 201203, China. E-mail: zhoumm368@163.com; Fax: +86 21 51322642; Tel: +86 2151322642

${ }^{b}$ Central Laboratory, Baoshan District Hospital of Integrated Traditional Chinese and Western Medicine of Shanghai, Shanghai University of Traditional Chinese Medicine, Shanghai 201999, China. E-mail: gouxiaojun1975@163.com; Fax: +86 21 36072150; Tel: +862156601100

${ }^{c}$ Department of Physiology, Shanghai University of Traditional Chinese Medicine, Shanghai 201203, China
${ }^{d}$ College of Chinese Pharmacy, Shanghai University of Traditional Chinese Medicine, Shanghai 201203, China

${ }^{e}$ College of Life Science, Qinghai Normal University, Xining, Qinghai, 810008, China ${ }^{f}$ Key Laboratory of Medicinal Animal and Plant Resources in Qinghai-Tibet Plateau, Xining, Qinghai, 810008, China

$\dagger$ Electronic supplementary information (ESI) available. See DOI: $10.1039 / \mathrm{c} 8 \mathrm{ra00074c}$

† Le Zhao and Zixu Zhang are co-first authors. 
effective antidepressants is very impressive. Chinese medicine is thought to be an effective way to find new antidepressants. ${ }^{4}$ Chlorogenic acid (3-O-caffeoylquinic acid, CGA) is not only a key constituent of coffee, which is considered as a beneficial substance for depression as its properties of antiinflammatory and antioxidant, ${ }^{5-7}$ but also an important active ingredient of many traditional Chinese medicines, like Eucommiae cortex (Duzhong), honeysuckle flower (Jinyinhua), etc. Studies have shown that CGA could reduce the production of a variety of pro-inflammatory cytokines, which is consistent with the neuroinflammatory hypotheses of depression and the effects of current antidepressant therapies. ${ }^{5-8}$ It was reported that CGA could promote 5-HT release through enhancing synapsin I expression, and orally administration of CGAenriched extract of Eucommia ulmoides Oliver (200, $400 \mathrm{mg}$ per $\mathrm{kg}$ per day) on mice for 7 days showed antidepressant-like effects in tail suspension test (TST). ${ }^{\mathbf{9 , 1 0}}$ Furthermore, according to a report of Park, ${ }^{\mathbf{1 1}}$ CGA isolated from Artemisia capillaris Thunb was showed antidepressant-like activity in mice with chronically restraint stress in forced swimming test (FST) and TST. It was indicated that CGA could cross the bloodcerebrospinal fluid barrier to display its neuron protection, ${ }^{\mathbf{1 0}}$ and even so, the anti-depressant efficacy of CGA is not certain.

The hypothalamic-pituitary-adrenal (HPA) axis is a major integrated system that maintains body homeostasis, which controls the stress responses of an individual. ${ }^{12}$ Stress triggers the release of corticotropin-releasing hormone (CRH), accompanied by that of adrenocorticotropic hormone (ACTH), and causes serotonergic system changes, such as increasing of the expression of 5-hydroxytryptamine $(5-\mathrm{HT})_{2 \mathrm{~A}}$ receptors. $^{\mathbf{1 3 , 1 4}}$ Chronically administrated ACTH increased the expression of 5$\mathrm{HT}_{2 \mathrm{~A}}$ receptor mRNA in the frontal cortex of rats, ${ }^{15}$ as well as blocked the antidepressant effects of the tricyclic antidepressants (imipramine), the noradrenaline reuptake inhibitor (desipramine), and the serotonin and noradrenaline reuptake inhibitor on the immobility time of FST (milnacipran). ${ }^{\mathbf{1 6 , 1 7}}$ Therefore, ACTH-treated rats might serve as an animal model of tricyclic anti-depressant treatment-resistant depression. Chronic ACTH-treatment (100 $\mu \mathrm{g}$ per rat per day, s.c., for 14 days) induced depressive-like behaviors in FST and open field test (OFT), which could be blocked by memantine (3,5dimethyladamantan-1-amine, $10 \mathrm{mg} \mathrm{kg}{ }^{-1}$, i.p.), , ${ }^{18}$ a noncompetitive NMDA receptor antagonist. Therefore, memantine was chose as positive control in this study.

Nowadays, metabolomics approach is a widely used tool for the discovery of biomarkers for disease diagnosis or prognosis. As to depression studies, metabolomics offers mechanistic insights into the potential pathophysiological features and assessments of the therapeutic effects of antidepressants. ${ }^{\mathbf{1 9 - 2 6}}$ Gas chromatography-mass spectrometry (GC-MS) has been widely applied in metabolomics studies as for its high sensitivity, high resolution and high reproducibility. ${ }^{22,23,25,26}$

In the present study, we firstly investigated whether CGA has antidepressant-like effects on chronic ACTH-treated rats using sucrose preference test (SPT) and OFT. Secondly, non-target urinary metabolomic strategy based on GC-MS detection was applied on the profiling of global metabolomic character of
ACTH-treated rats as well as ACTH-treated rats administrated with memantine and CGA. Finally, combined with pattern recognition and bioinformatics analysis methods, differential metabolites among each group and related possible metabolic pathways or biomarkers were illustrated. Our studies would provide new insights in the biology and pathology of depression disease, and also assess the antidepressant-like effects of CGA on tricyclic anti-depressant treatment-resistant rat model as well as obtain its potential therapeutic targets for future studies of the underlying biological mechanisms.

\section{Experimental procedures}

\section{Animal model and behavioral tests}

32 male Wistar rats (Shanghai Sippr-BK laboratory animal Co. Ltd., China) of 10 weeks weighs $230-250 \mathrm{~g}$ were randomly divided into control (Normal diet, $\mathrm{N}, n=8$ ) group and ACTHtreated group $(n=24)$ after one-week's adaption. The rats were kept in a standard laboratory environment with free access to food and water $\left(23{ }^{\circ} \mathrm{C}, 12 \mathrm{~h}\right.$ daylight cycles, and $55 \pm 15 \%$ humidity). All the procedures dealing with the animals in this study were performed in accordance with the Guidelines for Care and Use of Laboratory Animals of Shanghai University of Traditional Chinese Medicine and approved by the Animal Ethics Committee of Shanghai.

ACTH-treated rats were assigned randomly into 3 groups ( $n$ $=8$ ), ACTH-treated model group (M), positive control group (memantine, $\mathrm{M}+\mathrm{Mem}$ ) and CGA intervened group ( $\mathrm{M}+\mathrm{CGA})$. Each rat in these three groups received s.c. injection of ACTH (Chengdu Kaijie Peptide Co., Chengdu, China) at a dose of 100 $\mu \mathrm{g}$ per rat for 14 days, and saline control group $(\mathrm{N})$ received saline injection of same volume for 14 days. CGA intervened rats received CGA (cas: 327-97-9, purity $\geq 98 \%$, HPLC method, extracted from Eucommia ulmoides Oliver bark, Sichuan Weikeqi Bio-tech Co. Ltd., Sichuan, China) i.g. at dose of $500 \mathrm{mg}$ $\mathrm{kg}^{-1}$, and memantine positive control group rats received memantine (Mem, Sigma, USA) i.g. at dose of $30 \mathrm{mg} \mathrm{kg}^{-1}$, saline control group and ACTH-treated model group received saline i.g. in same volume. For SPT, ${ }^{27,28}$ rats were first trained to adapt $1 \%(\mathrm{v} / \mathrm{v})$ sucrose solution kept in two bottles in each cage for $72 \mathrm{~h}$ on day $15^{\text {th }}-17^{\text {th }}$. Then rats were deprived of water and food for a further $24 \mathrm{~h}$. Finally, rats were housed in individual cages with free access to two bottles containing $100 \mathrm{~mL}$ of sucrose solution $(1 \% \mathrm{w} / \mathrm{v})$ and water, and the volume of sucrose solution and water consumed within 1 hour was recorded. Sucrose preference was calculated as follows: sucrose consumption (\%) $=$ sucrose consumption $/($ water + sucrose consumption $) \times 100$. OFT as employed previously was used to define locomotor activity and evaluate the exploratory activity of animal on day $14^{\text {th }} \cdot{ }^{27}$ Open field arena used was an acrylic box $(77 \mathrm{~cm} \times 77 \mathrm{~cm}$ $\times 40 \mathrm{~cm}$ ) with the floor divided into 49 equal squares. Scores of crossing, rearing, and grooming were counted in a $6 \mathrm{~min}$ session. The box was cleaned with $10 \%$ ethanol between trials. After the last test, all rats were put into metabolic cages for $12 \mathrm{~h}$ urine sample collection, and then sacrificed by chloral hydrate (300 $\mathrm{mg} \mathrm{kg}^{-1}$ bodyweight, i.p.) anaesthesia for abdominal aorta blood collection. Blood samples were centrifuged at $3000 \times g$ 
and $4{ }^{\circ} \mathrm{C}$ for $15 \mathrm{~min}$, and the urine samples were centrifuged at $1500 \times g$ for $5 \mathrm{~min}$. The supernatant was collected and stored at $-80{ }^{\circ} \mathrm{C}$ for later analysis.

\section{Serum biochemical analysis}

Serum samples obtained from rats of four groups were measured for 5-HT, ACTH, CRH, dopamine (DA). These parameters were quantified using the corresponding commercial kits according to manufacturer's protocols (Cusabio Biotechnology Co., Ltd, Wuhan, China).

\section{Metabolomics analysis}

Urine samples were prepared for GC-MS analysis and relevant spectral acquisitions were performed according to our previously published methods with minor modifications. ${ }^{29,30}$ After being centrifuged at $12000 \mathrm{rpm}$ for $10 \mathrm{~min}, 100 \mu \mathrm{L}$ supernatant was extracted from urine sample. The degradation of urea was carried out by adding $70 \mathrm{IU}$ urease $\left(37^{\circ} \mathrm{C}, 15 \mathrm{~min}\right)$. The sample was derivatized with ethyl chloroformate, and L-2-chlorophenylalanine was used as an internal standard to maintain batch reproducibility. The sample analysis was carried out with GC-TOFMS (Pegasus HT, Leco Corp., St. Joseph, MI; electron ionization mode). One QC sample and one blank vial were run after each 10 samples. One- $\mu \mathrm{L}$ aliquot of analyte was injected with a splitless mode into DB-5MS capillary column coated with 5\% diphenyl cross-linked 95\% dimethylpolysiloxane $(30 \mathrm{~m} \times$ $250 \mu \mathrm{m}$ i.d., $0.25 \mathrm{~m}$ film; Agilent J\&W Scientific, Folsom, CA) for sample separation with helium as the carrier gas at constant flow rate of $1.0 \mathrm{~mL} \mathrm{~min}^{-1}$. The temperature of injection, transfer interface, and ion source was maintained at 270, 260, and $200{ }^{\circ} \mathrm{C}$, respectively. Temperature programming for $\mathrm{GC}$ was set to $2 \mathrm{~min}$ isothermal heating at $80{ }^{\circ} \mathrm{C}$, followed by $10{ }^{\circ} \mathrm{C} \mathrm{min} \operatorname{mov}^{-1}$ oven temperature ramp to $180{ }^{\circ} \mathrm{C}, 5{ }^{\circ} \mathrm{C} \min ^{-1}$ to $240^{\circ} \mathrm{C}$, and $25^{\circ} \mathrm{C} \mathrm{min}^{-1}$ to $290^{\circ} \mathrm{C}$, and a final 9 min maintained temperature at $290^{\circ} \mathrm{C}$. Electron impact ionization $(70 \mathrm{eV})$ at full scan mode ( $\mathrm{m} / \mathrm{z} 30-600)$ was used, with an acquisition rate of 20 spectra per $\mathrm{s}$ in TOFMS setting.

The peak information acquired from GC-TOFMS analysis was exported in NetCDF format by ChromaTOF software (v4.44, Leco Co., Los Angeles, CA), and further pretreated by R-2.13.2 (Lucent Technologies). The final data matrix was constructed with normalized peak areas with two vectors: sample names as observations in the first column, and retention times/peaks as the response variables in the first row. ${ }^{31}$ Principal component analysis (PCA) ${ }^{32}$ and partial least squares projection to latent structures and discriminant analysis (PLS-DA) ${ }^{33}$ were employed to process the acquired three-dimensional matrix for multivariate analysis with SIMCA-P 11 software (Umetrics, Umeå, Sweden). Samples from the same groups were classified into one for PLS-DA modeling. The results of PCA and PLS-DA were displayed as scores plots that visualized the clustering of the samples and indicated the similarity of samples. The closer clustering of the samples represented higher compositional similarity, whereas the further clustering represented diverse metabolomic composition. The purpose of PLS-DA was to calculate models differentiating groups and identify the response variables contributing most to the model and further identify potential markers. Orthogonal projection to latent structure with discriminant analysis (OPLS-DA) was used for predicting or evaluating variations in frame areas between groups. The corresponding $V$-plot has been established and the Variable Importance Parameter (VIP) values summarized the overall contribution of each $X$-variable to the model. ${ }^{34,35}$

\section{Statistical analysis}

SPSS 16.0 was used to analysis the data from behavioral tests. All values are expressed as the mean \pm standard errors of the mean (SEM). Statistical significance among the groups was conducted by one-way analysis of variance (ANOVA). In all cases, probability values of less than 0.05 were considered to show a statistical significance. Statistical analyses were performed using Graphpad Prism 6.0 (GraphPad Software Inc., USA).

\section{Results}

\section{Behavioral analysis}

To investigate the effects of CGA on ACTH-treated rats, we performed SPT (Fig. 1) and OFT (Fig. 2). In general, SPT has been used to evaluate stress-induced anhedonia. ${ }^{36}$ A lower percentage of sucrose preference was observed in $\mathrm{M}$ group when compared with $\mathrm{N}$ group (Fig. $1, P<0.001$ ). After drug treatments, sucrose preference dramatically increased in $\mathrm{M}+\mathrm{Mem}$ $(P<0.01)$ and $\mathbf{M}+$ CGA group (Fig. $1, P<0.05)$. The scores of crossing, rearing, and grooming were presented in Fig. 2. As expected, $\mathbf{M}$ group significantly decreased the scores of crossing $(P<0.01)$, rearing $(P<0.001)$, and grooming $(P<0.001)$, when compared with $\mathrm{N}$ group. $\mathrm{M}+$ Mem group enhanced the scores of crossing $(P<0.05)$, rearing $(P<0.01)$, and grooming $(P<0.05)$. The administration of CGA $500 \mathrm{mg} \mathrm{kg}^{-1}$ for 14 days increased the scores of crossing $(P<0.05)$, rearing $(P<0.05)$, but showed no effect on the scores of grooming (Fig. 2).

\section{The effect of CGA on serum biochemical parameters}

Serum biochemical parameters of 5-HT, ACTH, CRH, DA were presented in Fig. 3. The serum concentrations of 5-HT and DA

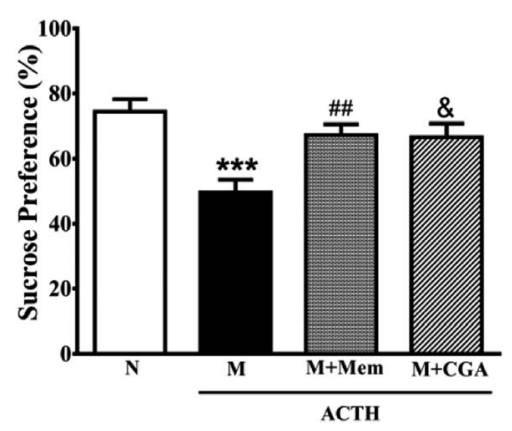

Fig. 1 Antidepressant effect of Mem and CGA on ACTH-treated rats in sucrose preference test. Data are shown as the mean \pm SEM. Significant differences between $M$ vs. $N$ are indicated as: $* * * P<0.001$; significant differences between $M+M e m$ vs. $M$ are indicated as: ${ }^{\# \#} P<$ 0.01 ; significant differences between $M+C G A$ vs. $M$ are indicated as: ${ }^{8} P<0.05$. 

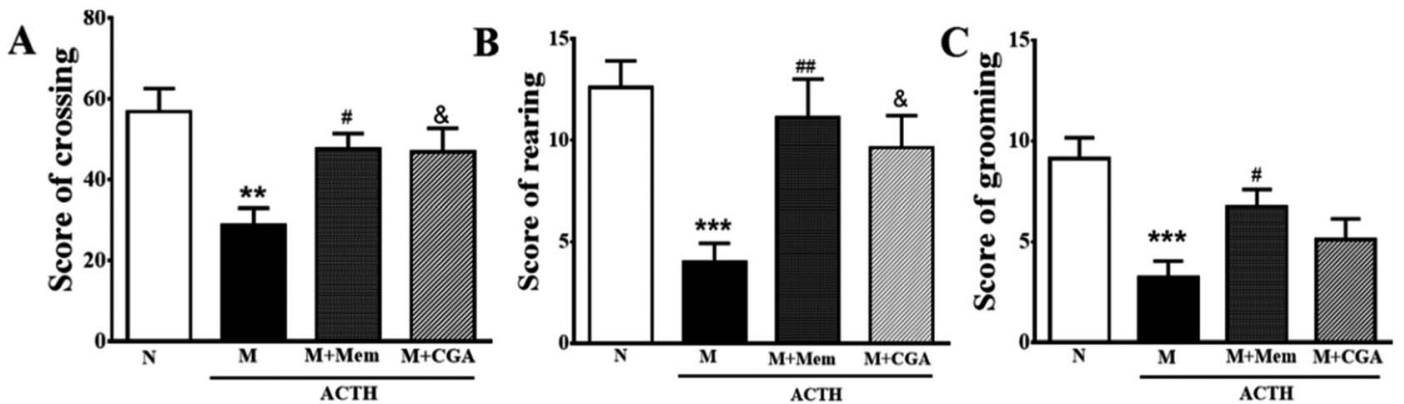

Fig. 2 Antidepressant effect of Mem and CGA on the spontaneous activity of ACTH-treated rats in open field experiment. (A) The scores of crossing, (B) the scores of rearing, and $(C)$ the scores of grooming were counted in a 6 min session for 14 days. Data are shown as the mean \pm SEM. Significant differences between $M$ vs. $N$ are indicated as: $* * P<0.01, * * * P<0.001$. Significant differences between $M+M e m$ vs. $M$ are indicated as: ${ }^{\#} P<0.05,{ }^{\# \#} P<0.01$. Significant differences between $M+C G A$ vs. $M$ are indicated as: ${ }^{8} P<0.05$.

were significantly decreased $(P<0.01)$, while the levels of ACTH and $\mathrm{CRH}$ were elevated in $\mathrm{M}$ group as compared to $\mathrm{N}$ group $(P<$ 0.01). After the treatment of CGA, the levels of 5-HT $(P<0.05)$ and DA $(P<0.01)$ were dramatically increased, along with the inhibited levels of ACTH $(P<0.05)$ and CRH $(P<0.01)$ in ACTHtreated rats.

\section{Multivariate statistical analysis}

In order to evaluate the effects of CGA on ACTH-induced depressant rat model, PCA and PLS-DA models were constructed to analyze the data obtained from GC-MS detection (Fig. 4 and S1 $\dagger$ ). The PCA score plots demonstrated significant differences between $\mathrm{N}$ and $\mathrm{M}$ groups $\left(R^{2} X=0.667, Q^{2}(\mathrm{cum})=\right.$ 0.466; Fig. $4 \mathrm{~A}$ and Table $\mathrm{S} 1 \dagger), \mathrm{M}$ and $\mathrm{M}+\operatorname{Mem}$ groups $\left(R^{2} X=\right.$
$0.645, Q^{2}$ (cum) $=0.347$; Fig. $\left.4 \mathrm{~B}\right), \mathrm{M}$ and $\mathrm{M}+\mathrm{CGA}$ groups $\left(R^{2} X=\right.$ $0.68, Q^{2}$ (cum) $=0.466$; Fig. 4C). Comparisons among the four groups were also performed using PCA and shown in Fig. 4D. N group was completely separated from $\mathrm{M}$ group, while $\mathrm{M}+\mathrm{Mem}$ and $\mathrm{M}+\mathrm{CGA}$ groups were all settled around $\mathrm{N}$ group. Then, PLSDA was carried out to maximize the difference of metabolic profiles among the four groups (Fig. S1 $\dagger$ ). The scores plot from PLS-DA model showed a clear separation between $\mathrm{N}$ and $\mathrm{M}$ groups $\left(R^{2} X=0.646, R^{2} Y=0.988, Q^{2}\right.$ (cum) $=0.955$; Fig. S1A and Table S1†), $\mathrm{M}$ and $\mathrm{M}+\mathrm{Mem}$ groups $\left(R^{2} X=0.705, R^{2} Y=\right.$ $0.998, Q^{2}(\mathrm{cum})=0.998$; Fig. S1B and Table S1 $\left.\dagger\right), \mathrm{M}$ and $\mathrm{M}+$ CGA groups $\left(R^{2} X=0.622, R^{2} Y=0.995, Q^{2}(\mathrm{cum})=0.967\right.$; Fig. S1C and Table S1 $\dagger$ ), indicating that CGA exerts effect on metabolism of ACTH-induced depressant rats. The parameters of $R^{2} X$ and $Q^{2}$ were employed for evaluation of PCA model, as
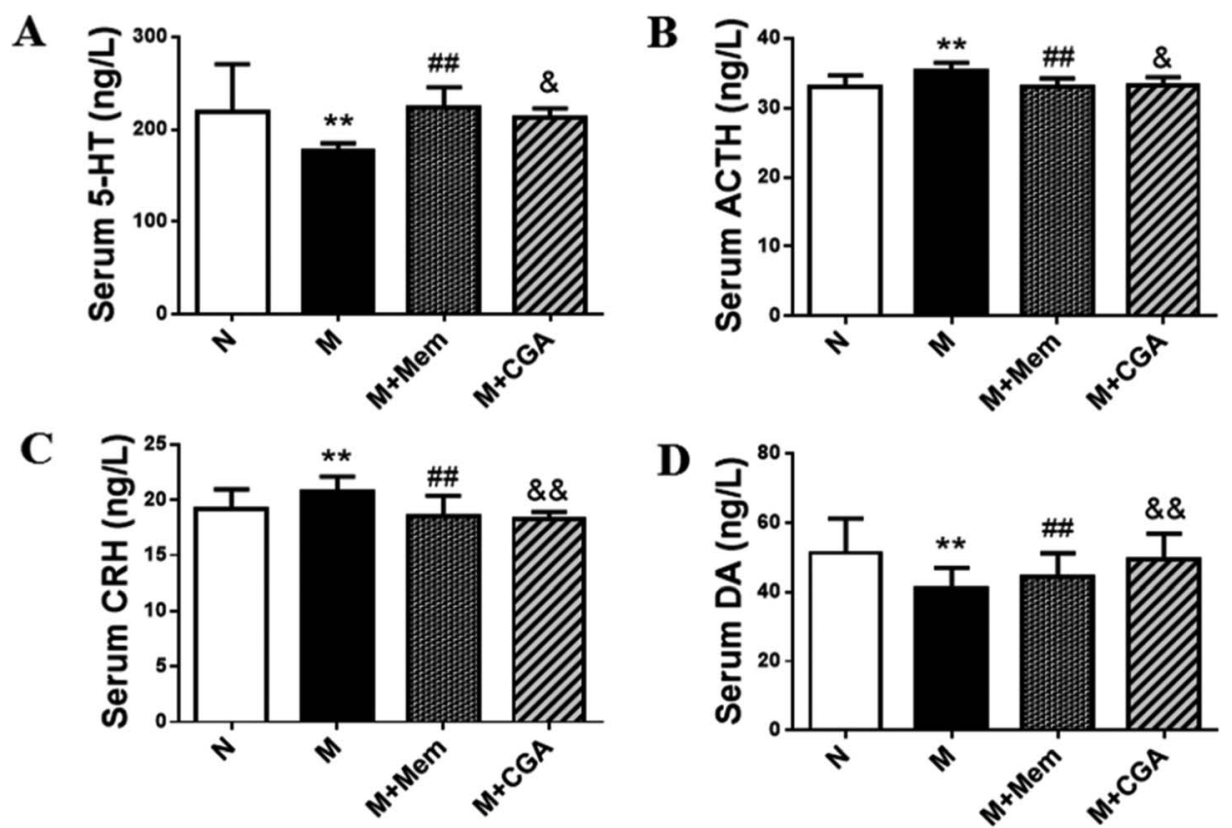

Fig. 3 Effects of CGA administration on serum biochemical parameters. The concentrations of (A) serum 5-HT, (B) serum ACTH, (C) serum CRH, (D) serum DA in four groups. Data are shown as the mean $\pm S D$. Significant differences between $M v s$. $N$ are indicated as: $* * P<0.01$. Significant differences between $M+M e m$ vs. $M$ are indicated as: ${ }^{\#} P<0.05,{ }^{\# \#} P<0.01$. Significant differences between $M+C G A$ vs. $M$ are indicated as: ${ }^{8} P<$ $0.05,{ }^{\text {\&ष }} P<0.01$. 
A

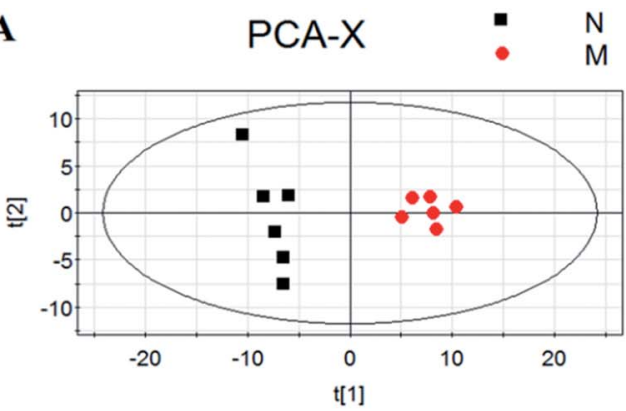

C
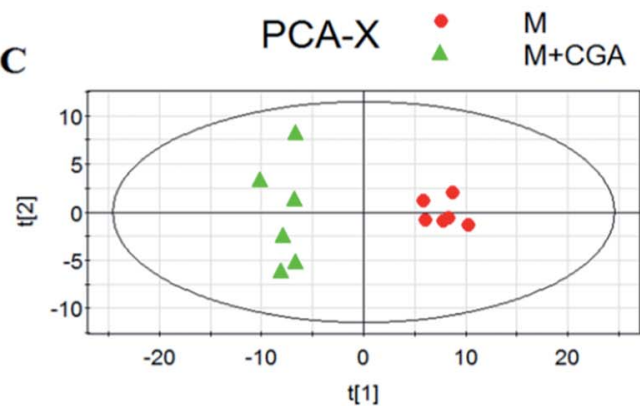
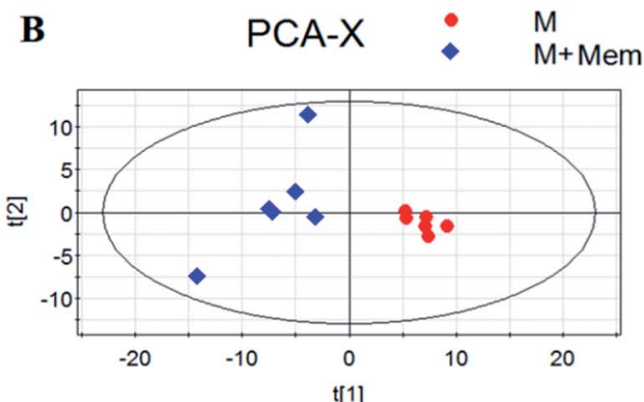

PCA-X

$\because \quad \begin{aligned} & N \\ & -\quad M \\ & M\end{aligned}$
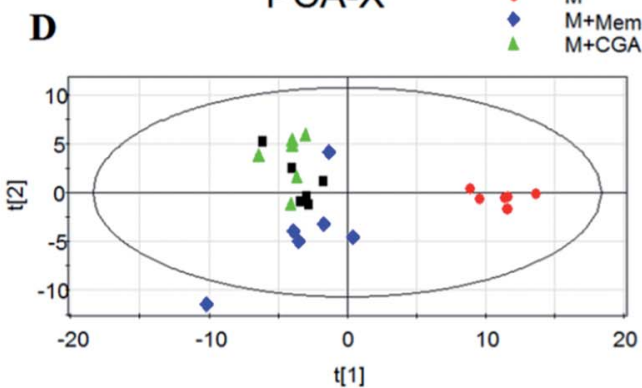

Fig. 4 Scores plots of multivariate statistical analysis on urine samples. (A) Partial least squares-discriminate analysis (PCA) scores plot of normal $(N)$ group versus model (M) group, (B) PCA scores plot of model (M) group versus memantine (M+Mem) group, (C) PCA scores plot of model (M) group versus chlorogenic acid (M + CGA) group, (D) PCA scores plot of N, M, M + Mem, M + CGA groups.

A

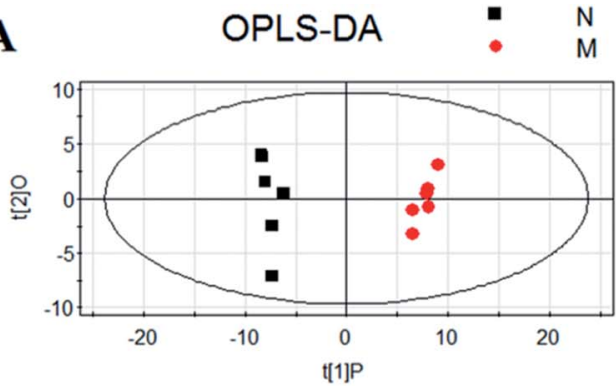

B

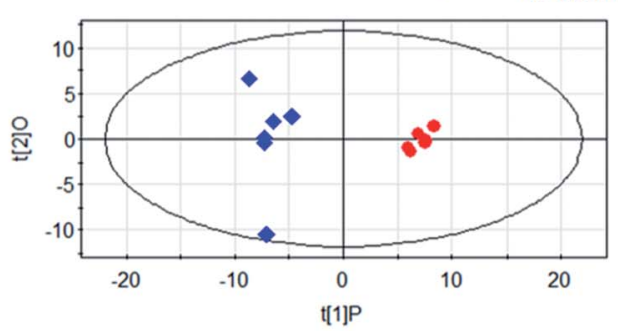

C
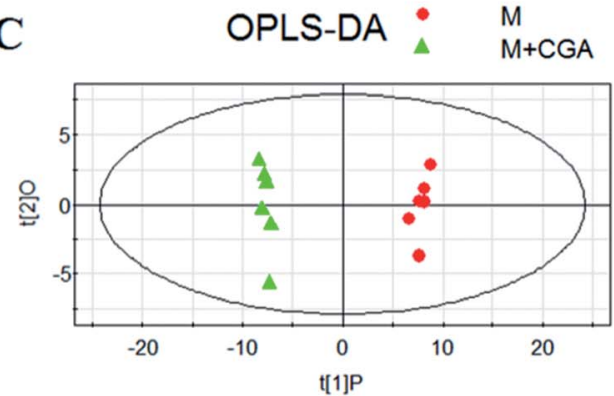

V-Plot

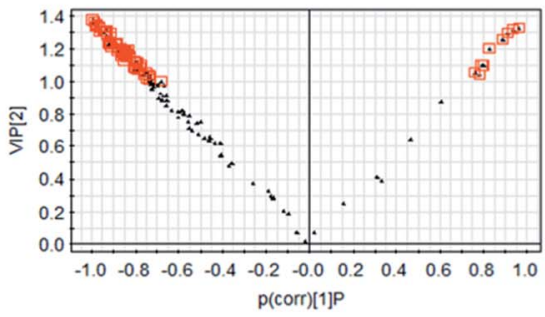

V-Plot

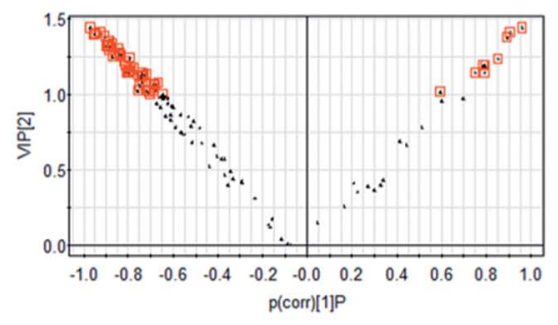

V-Plot

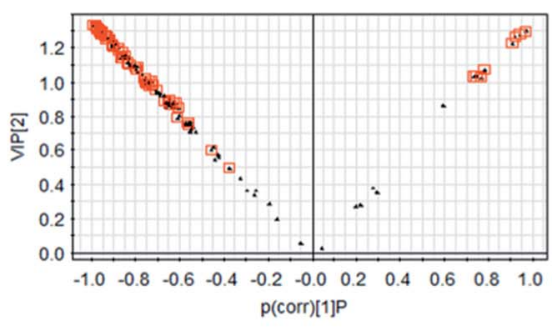

Fig. 5 Orthogonal partial least squares-discriminant analysis (OPLS-DA) score plots (left) and corresponding loadings plots (right), derived from metabolites of (A) M group against those of $N$ group, (B) $M+M e m$ group against those of $M$ group, and (C) $M+C G A$ group against those of $M$ group. 


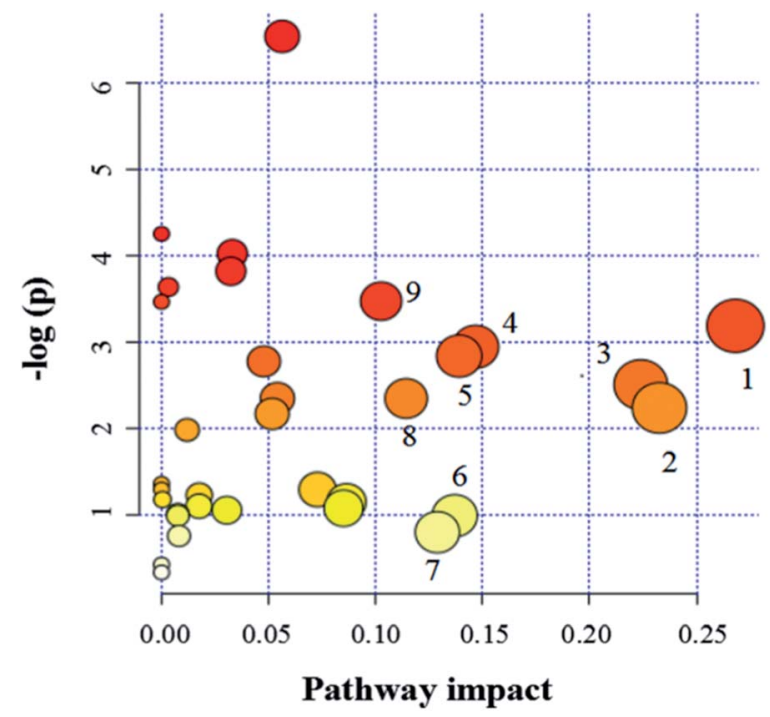

Fig. 6 Pathway analysis of metabolites. (1) Beta-alanine metabolism, (2) glycine, serine and threonine metabolism, (3) galactose metabolism, (4) pyruvate metabolism, (5) arginine and proline metabolism, (6) inositol phosphate metabolism, (7) glyoxylate and dicarboxylate metabolism, (8) ascorbate and aldarate metabolism, (9) alanine, aspartate and glutamate metabolism.

well as $R^{2} X, R^{2} Y$ and $Q^{2}$ (cum) were used for evaluation of PLSDA model. $R^{2} X$ and $R^{2} Y$ were usually used to quantify the goodness-of-fit of model and $Q^{2} Y$ was employed to assess model predictability. ${ }^{37}$ Generally, the values of $R^{2} Y$ and $Q^{2}$ above 0.8 were considered as excellent models. According to the parameters $\left(R^{2} X, R^{2} Y\right.$ and $\left.Q^{2}\right)$ shown in ESI Table S1, $\dagger$ the PLS-DA model was positive and valid.

\section{Key metabolites analysis}

In order to find the changed metabolites in urine samples between $\mathrm{N}$ and $\mathrm{M}$ groups, $\mathrm{M}$ and $\mathrm{M}+\mathrm{Mem}$ groups, $\mathrm{M}$ and $\mathrm{M}+$ CGA groups, the supervised OPLS-DA and corresponding $V$-plot were constructed (Fig. 5). Variable importance for the projection (VIP) values exceeding 1.0 were calculated and subsequently analyzed by paired $t$-test analysis (Table $\mathrm{S} 2 \dagger$ ). The metabolites in the level of significance difference $(P<0.05)$ were considered as potential biomarkers, which were responsible for the difference between $\mathrm{N}$ and $\mathrm{M}$ group. A total of nineteen metabolites were identified as potential biomarkers for chronically ACTHinduced depression. The heat map of differential metabolites was showed in Fig. 7, in which the relative changes of these metabolites might be readily observed. The results showed that the decreased levels of alanine (beta-alanine), propanedioic acid, threonine, serine, galactose, myo-inositol, D-lactic acid, acetic acid, urea, creatinine, proline, asparagine, kynurenic acid, along with the increased levels of hippurate and ferulic acid in $\mathrm{M}$ group compared with $\mathrm{N}$ group (Table S2; $\dagger$ Fig. 7). The levels of beta-alanine, propanedioic acid, threonine, serine, myo-inositol, acetic acid, creatinine, alanine, kynurenic acid were increased while hippurate and ferulic acid were decreased after Mem or CGA treatment, and these altered metabolites could be associated with the antidepressant effect of CGA (Table S2;† Fig. 7).

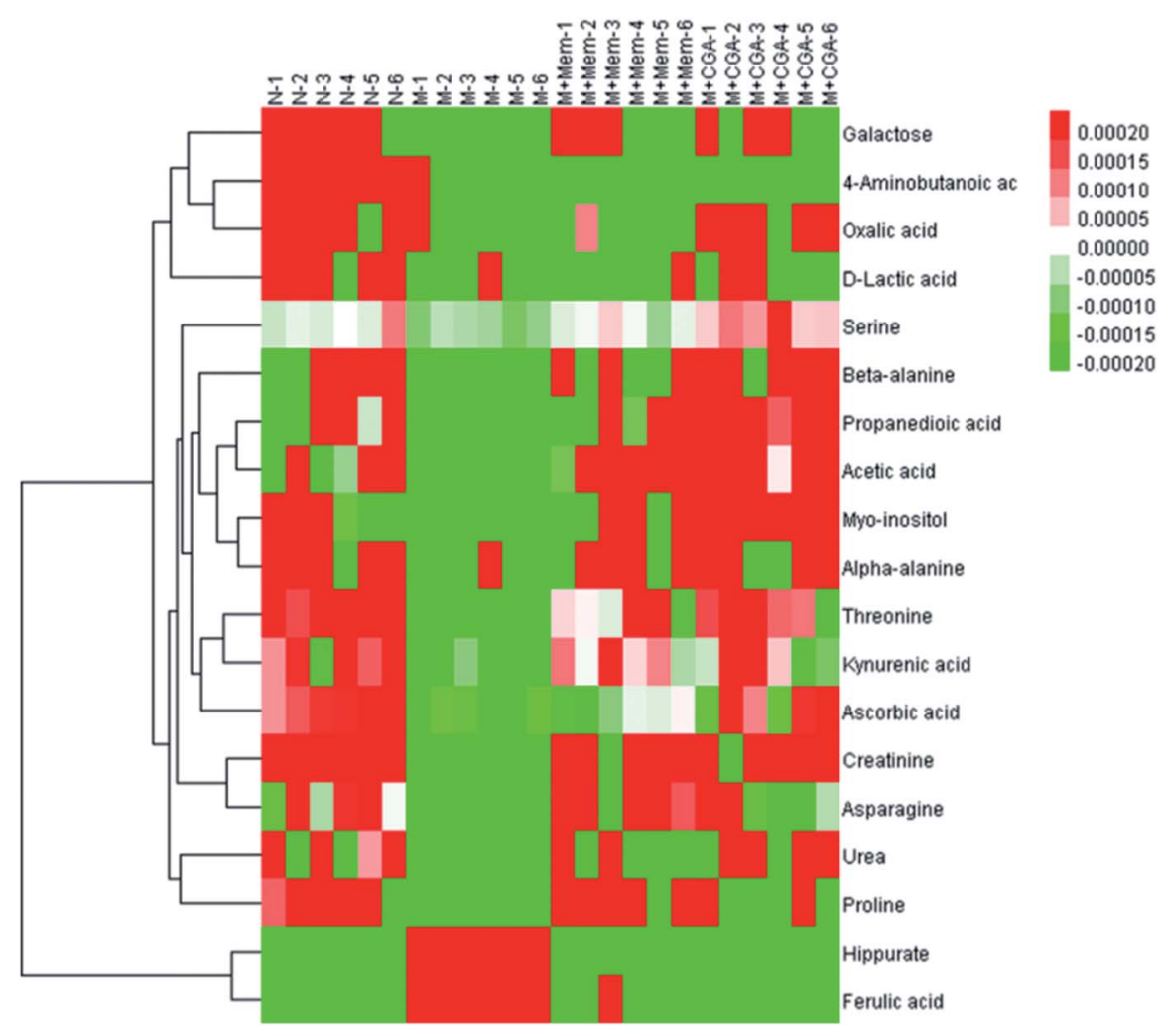

Fig. 7 Heat map of the differential metabolites in N, M, M + Mem and M + CGA groups. 
Table 1 List of significant metabolites accountable for class discrimination ${ }^{a}$

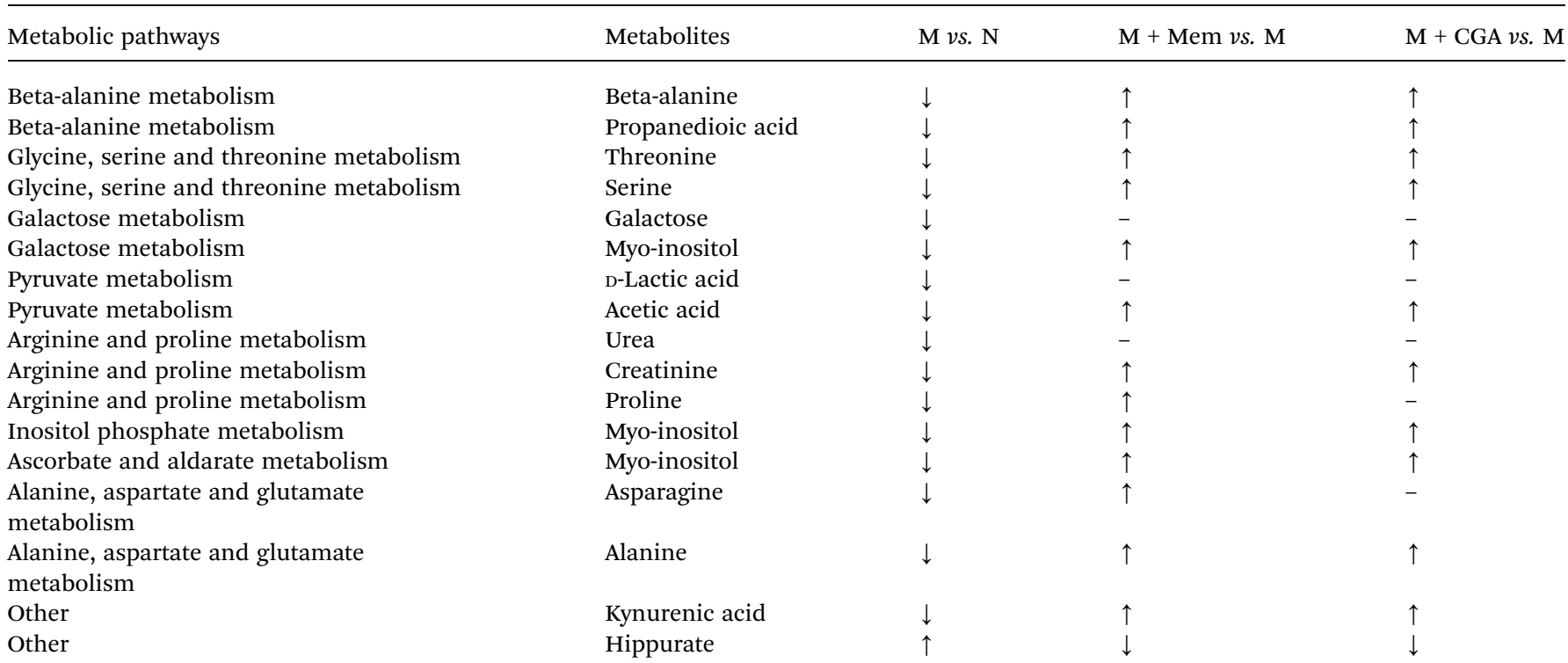

${ }^{a}$ These metabolites are verified by reference compounds available. The short dash line (-) indicates no significant variation. The arrow denotes the VIP value greater than 1.0 and the up- (or down-) regulation of the arrow represents the relative increased (or decreased) concentration.

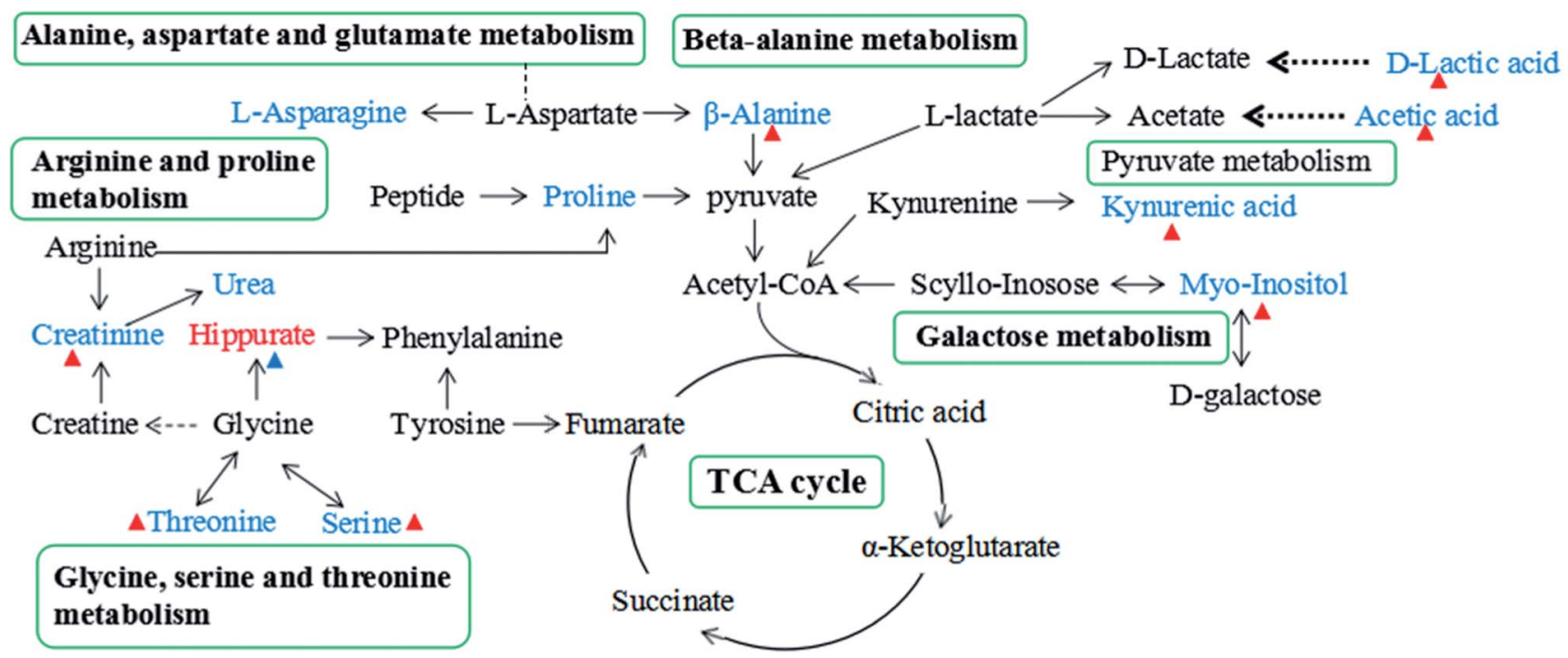

Fig. 8 The overview of metabolic pathways related to the CGA treatment on ACTH-induced depression in urine. Red-labelled metabolites indicate up-regulation in ACTH-treated rats, while blue-labeled metabolites represent the down-regulation compared with the normal rats. The red triangles $(\Delta)$ represent metabolites with increased levels in the $M+C G A$ group, blue triangles $(\Delta)$ represent metabolites with decreased levels in the $M+C G A$ group when compared with the $M$ group.

\section{Metabolic pathway analysis}

Since enzymes or metabolites involved in the same biological processes are often changed together, ${ }^{38}$ Pathway-based metabolomics features are analogues of metabolomics biomarkers that provide more information on biological functions. ${ }^{39}$ Based on the overall 19 biomarkers identified from $\mathrm{M}$ versus $\mathrm{N}$ groups, MetaboAnalyst 3.0 was used to identify perturbed metabolic pathways (Table 1 and Fig. 6). ${ }^{40}$ Kyoto Encyclopedia of Genes and Genomes (KEGG; http://www.genome.jp/kegg/) and Human Metabolome Database (HMDB; http://www.hmdb.ca/) were used for confirmation. The results showed that there were nine dramatically affected metabolic pathways (impact value $>0.10$ ), including beta-alanine metabolism, glycine, serine and threonine metabolism, galactose metabolism, pyruvate metabolism, arginine and proline metabolism, inositol phosphate metabolism, glyoxylate and dicarboxylate metabolism, ascorbate and aldarate metabolism, alanine, aspartate and glutamate metabolism and other metabolic pathways (Nod color and size are indicative of $P$-value significance and pathway impact, respectively, Fig. 6). Based on the relationships among the metabolic 
biomarkers, we summarized the pathways into a brief plot that contained most of the altered metabolites (Fig. 8).

\section{Discussion}

Several previous studies have confirmed that chronic treatment of ACTH could induce depression-like behaviour. ${ }^{17,41}$ In the present study, the OFT and SPT were measured during the experimental period to evaluate the antidepressant-like effect of CGA in the ACTH-induced depression model. The results showed that Mem and CGA exerted antidepressant-like effects on SPT and OFT in ACTH-treated rats. In addition, ACTHinduced depressive rats showed a lower level of 5-HT and DA, as well as a higher level of ACTH and CRH. As a consequence of CGA treatment, changes in the levels of 5-HT, ACTH, $\mathrm{CRH}$, and DA indicated alterations in the HPA axis function. ${ }^{42}$ Urinary samples are more convenient for collecting when compared with blood samples, especially for small animals. Furthermore, noninvasive sampling could avoid interference in the neuropsychological activities of animals. Most important of all, they present the combination of metabolites in a period of time (e.g. $24 \mathrm{~h}$ ), while the biomarkers in blood have a relatively shorter window period. ${ }^{43}$ So far, urine has been widely used in animal or human metabolomics studies to diagnose disease and serve as an early warning in preclinical stages. $^{\text {44-47 }}$ Therefore, our study employed GC-MS-based metabolomics to study the urinary metabolic phenotypes between normal and depressed rats and identify the potential biomarkers. The concentrations of the endogenous metabolites, alanine, propanedioic acid, threonine, serine, myoinositol, acetic acid, creatinine, hippurate, ferulic acid, and kynurenic acid were dramatically changed after the treatment of CGA in ACTH-induced depressant rats' urine. These metabolites might be the biomarkers related to the efficacy of CGA.

\section{Serum biochemical parameters analysis}

Serotonin (5-HT) has been reported to play a vital role in the pathogenesis of depression. ${ }^{48} 5$-HT, monoamine neurotransmitter metabolites existed in the hippocampus, showed a significant lower level in $\mathrm{M}$ group compared with $\mathrm{N}$ group. Decreased 5-HT level is associated with the development of depression. Furthermore, our study also found that CGA could significantly improve 5-HT level in serum. The serum ACTH level was markedly increased in $\mathbf{M}$ group. It was reported that rats treated with ACTH could be served as an animal model of depression. ${ }^{16}$ After the treatment of Mem and CGA, the level of ACTH was significantly decreased. CRH has been reported to be related to increase depressive symptoms. ${ }^{49}$ The lower levels of CRH in $\mathbf{M}+$ Mem and $\mathbf{M}+$ CGA group might be related to the alleviation of depression. The interaction of DA with the limbic system is likely to be associated with stress and depression. ${ }^{50}$ The monoamine theory assumes that depression is related to low levels of monoamine neurotransmitters, especially 5-HT and dopamine. ${ }^{51}$ Compared with $\mathrm{M}$ group, our results showed that DA content was higher in $\mathbf{M}+\mathrm{Mem}$ and $\mathbf{M}+$ CGA group. In conclusion, these results suggest that the changes in the ACTHinduced depressive rats might play a specific role in the development of depression. Mem and CGA treatments could reverse the changes in ACTH-treated rats and exert antidepressant-like effects.

\section{Changes in energy metabolism}

Beta-alanine metabolism might be one of the most affected metabolic pathways. Compared with $\mathrm{N}$ group, levels of betaalanine and propanedioic acid were all decreased in $\mathbf{M}$ group. Asparagine and alanine involved in the pathway of alanine, aspartate and glutamate metabolism might be tightly related with the depression. Aspartate and alpha-alanine were significantly decreased in urine samples from depressive rats compared with $\mathrm{N}$ group, while only alanine was significantly reversed after CGA treatment.

Alanine, a nonessential amino acid, is one of the most crucial amino acids released by muscle. ${ }^{52}$ Meanwhile, it is an inhibitory neurotransmitter in the brain and an important participant and regulator in glucose metabolism. ${ }^{53}$ As a major energy source, alanine might enter tricarboxylic acid (TCA) cycle and glucose in gluconeogenesis. ${ }^{54} \beta$-alanine, probably as a neuromodulator, occurs naturally in the human central nervous system (CNS). ${ }^{55} \beta$-alanine plays an essential role in $\beta$-alanine metabolism pathway, which are tightly associated with the genes of hormones, cytokines and neurotransmitters. ${ }^{56}$ Aspartate, derived from the TCA cycle, is involved in energy production. Alanine and aspartate have a great impact on alanine, aspartate and glutamate metabolism pathway. The relatively higher level of alanine in the urine of CGA-treated ACTHinduced depressive rats might be related to the promotion of alanine metabolism for energy supply.

\section{Amino acid metabolism}

Pyruvate, arginine and proline metabolism. D-Lactic acid, acetic acid, proline and creatinine were dramatically reduced in $\mathbf{M}$ group compared with $\mathrm{N}$ group. These statistically significant perturbations are involved in pyruvate, arginine and proline metabolism. Pyruvate is crucial for mitochondrial ATP generation and it could drive for multiple biosynthetic pathways intersecting the TCA cycle. Aberrant pyruvate metabolism plays a prominent role in neurodegenerative disorders. ${ }^{57}$ Lower levels of D-lactic acid and acetic acid were observed in $\mathrm{M}$ group when compared with $\mathrm{N}$ group. However, compared with $\mathrm{M}$ group, the level of acetic acid was increased both in $\mathrm{M}+\mathrm{Mem}$ and $\mathrm{M}+\mathrm{CGA}$ group. Acetic acid has been reported to affect some critical metabolic pathways, such as the pathway of glyoxylate and TCA cycle. ${ }^{58}$ The degradative products of lactic acid were acetic acid and 1,2-propanediol. ${ }^{59}$ The content of acetic acid was found to be relatively higher in $\mathbf{M}+$ CGA group than that of $\mathbf{M}$ group, which might promote the pathway of pyruvate metabolism.

Proline, a precursor of pyruvate, is widely distributed in the CNS. $^{60}$ The higher level of proline in $\mathbf{M}+$ Mem group than in $\mathbf{M}$ group indicated that Mem might activate the biosynthesis of pyruvate. The lower level of proline in $\mathbf{M}$ group might be involved in the pathogenesis of ACTH-induced depression. 
Creatinine, a nonenzymatic breakdown product creatine phosphate, is crucial for cellular energy transportation. ${ }^{61}$ The decreased level of creatinine in $\mathrm{M}$ group might indicate altered energy metabolism. Energy deficiency is one of the most commonly represented depressive symptoms in major depressive disorder. ${ }^{62}$ The content of creatinine was dramatically increased after the treatment of CGA and it would be helpful in evaluating the efficacy of CGA on the treatment of depression.

Glycine, serine and threonine metabolism. The pathway of glycine, serine and threonine metabolism might have great impact on the progress of depression disease. Serine and threonine were dramatically decreased in $\mathrm{M}$ group compared with $\mathrm{N}$ group. Previous studies suggested that serine modulated behaviors associated with depression, such as reduced immobility in FST. ${ }^{63}$ Elevated serine concentrations in M + CGA group might lead to a depression-protected phenotype in rats. Threonine is an indispensable amino acid and was lower in $\mathrm{M}$ group compared with $\mathrm{N}$ group.

Our work found that serine and threonine played vital roles in the pathway of glycine, serine and threonine metabolism and were markedly decreased in $\mathrm{M}$ group compared with $\mathrm{N}$ group. Serine and threonine, belonging to the amino acids, were closely related to potential anti-oxidation. Additionally, compared to $\mathrm{M}$ group, the contents of serine and threonine were elevated in $\mathrm{M}+\mathrm{Mem}$ and $\mathrm{M}+\mathrm{CGA}$, which might be a factor associated with the treatment of depression.

Galactose metabolism, inositol phosphate metabolism and other metabolism. Galactose, a reducing sugar, could be transformed into hydrogen peroxide and aldose via the action of galactose oxidase, leading to the formation of an oxygen-derived free radicals and a superoxide anion. ${ }^{64}$ Myo-inositol plays a vital role in the phosphoinositide secondary messenger pathway. ${ }^{65}$ These two metabolites were responsible for galactose metabolism and were decreased in depressive rats. An elevated level of myo-inositol was observed in $\mathrm{M}+\mathrm{Mem}$ and $\mathrm{M}+\mathrm{CGA}$ group when compared with $\mathrm{M}$ group. Moreover, myo-inositol is an important participant and regulator in inositol phosphate metabolism. The significantly changed myo-inositol level after CGA treatment indicated that CGA could affect depression by regulating the metabolism of galactose and inositol phosphate. However, the underlying molecular mechanism of myo-inositol in the pathophysiology of depression is still unclear.

Hippurate, a common component of urine and an acyl glycine, is the metabolite of phenylalanine produced by combinatorial metabolism between the host and the gut microbiome. ${ }^{66}$ The significantly changed hippurate level after CGA treatment suggested that CGA might affect depression through the combinatorial metabolism between the host and the gut microbiome.

In all, CGA produces an antidepressant-like effect in chronic ACTH-treated rats by regulating nine perturbed metabolic pathways and their related metabolites.

\section{Conclusion}

As a brain disorder formed by the interaction of diversely heterogeneous pathogenic mechanisms, depression is now recognized as a systemic disease. Metabolomics is a powerful tool to study the mechanism of disease from a holistic perspective. In this study, differences between normal and chronic ACTH-treated rats were observed in behavioural and metabolic patterns. With global urinary metabolomics analysis method as our previously studies, ${ }^{29,30} 19$ differential metabolites were identified in ACTH-treated rats. Meanwhile, 12 out of 19 differential metabolites were reversed with CGA intervention. Furthermore, combined with pattern recognition and bioinformatics analysis, nine metabolic pathways were identified as perturbed metabolic pathways. These results would be helpful in understanding the pathophysiological mechanism of chronic ACTH-induced rat depressant model, and evaluating the efficacy of CGA on the treatment of depression. In conclusion, our work shows that metabolomics method combined with pattern recognition and network analysis is a promising strategy to explore the pathophysiological mechanism of depression, and evaluate the intervention of xenobiotics.

\section{Conflicts of interest}

The authors declare that they have no competing interests.

\section{Abbreviations}

$\begin{array}{ll}\text { ACTH: } & \text { Adrenocorticotropic hormone } \\ \text { CGA: } & \text { Chlorogenic acid } \\ \text { CRH: } & \text { Corticotropin-releasing hormone } \\ \text { DA: } & \text { Dopamine } \\ \text { FST: } & \text { Forced swimming test } \\ \text { GC/MS: } & \text { Gas chromatography coupled to mass spectrometry } \\ \text { HPA: } & \text { Hypothalamic-pituitary-adrenal } \\ \text { MDD: } & \text { Major depressive disorder } \\ \text { OFT: } & \text { Open field test } \\ \text { OPLS- } & \text { Orthogonal projections to latent structures } \\ \text { DA: } & \text { discriminant analysis } \\ \text { PCA: } & \text { Principal component analysis } \\ \text { PLS-DA: } & \text { Partial least squares discriminate analysis } \\ \text { SPT: } & \text { Sucrose preference test } \\ \text { TCA: } & \text { Tricarboxylic acid } \\ \text { TST: } & \text { Tail suspension test } \\ \text { 5-HT: } & \text { 5-Hydroxytryptamine }\end{array}$

\section{Acknowledgements}

This study was financially supported by National Natural Science Foundation of China (No. 81473475), the innovation project of Shanghai University of TCM (No. ZYX-CXYJ-018), Shanghai Municipal Education Committee (No. 2013JW17), Key Laboratory of Medicinal Animal and Plant Resources in Qinghai-Tibet Plateau, Xining, Qinghai, China (No. 2017-z-y25), and further accelerating the development of Chinese medicine three-year action plan of Shanghai (2014-2016) (No: ZY3-RCPY3-1013). 


\section{References}

1 H. A. Sackeim, J. Clin. Psychiatry, 2001, 62(suppl. 16), 10-17.

2 H. Jick, J. A. Kaye and S. S. Jick, J. Am. Med. Assoc, 2004, 292(3), 338-343.

3 S. Maeng and C. A. Jr Zarate, Curr. Psychiatry Rep., 2007, 9(6), 467-474.

4 J. Gong, F. Yin, Y. Hou and Y. Yin, Can. J. Anim. Sci., 2014, 94, 223-241.

5 S. Hall, B. Desbrow, S. Anoopkumar-Dukie, A. K. Davey, D. Arora, C. McDermott, M. M. Schubert, A. V. Perkins, M. J. Kiefel and G. D. Grant, Food Res. Int., 2015, 76, 626-636.

6 Z. Ruan, S. M. Mi, L. L. Zhou, Y. Zhou, J. Li, W. H. Liu and Z. Y. Deng, J. Funct. Foods, 2016, 26, 698-708.

7 Y. Zhou, Z. Ruan, L. Zhou, X. Shu, X. Sun, S. Mi, Y. Yang and Y. Yin, Biochem. Biophys. Res. Commun., 2016, 469(4), 10831089.

8 W. Shen, R. Qi, J. Zhang, Z. Wang, H. Wang, C. Hu, Y. Zhao, M. Bie, Y. Wang, Y. Fu, M. Chen and D. Lu, Brain Res. Bull., 2012, 88(5), 487-494.

9 Y. Zhou, Z. Ruan, X. L. Li, S. M. Mi, M. Jiang, W. H. Liu, H. S. Yang, X. Wu, G. L. Jiang and Y. L. Yin, J. Anim. Sci., 2016, 94, 164-168.

10 J. Wu, H. Chen, H. Li, Y. Tang, L. Yang, S. Cao and D. Qin, Molecules, 2016, 21(3), 260.

11 S. Park, Y. Sima and P. Hanb, Anim. Cells Syst., 2010, 14, 253259.

12 N. Hohne, M. Poidinger, F. Merz, H. Pfister, T. Brückl, P. Zimmermann, M. Uhr, F. Holsboer and M. Ising, Biol. Psychol., 2014, 103, 267-275.

13 B. E. Leonard, Eur. Psychiatry, 2005, (suppl. 3), S302-S306.

14 G. N. Pandey, Y. Dwivedi, H. S. Rizavi, X. Ren, S. C. Pandey, C. Pesold, R. C. Roberts, R. R. Conley and C. A. Tamminga, Am. J. Psychiatry, 2002, 159(3), 419-429.

15 Y. Kitamura, Y. Fujitani, K. Kitagawa, T. Miyazaki, H. Sagara, H. Kawasaki, K. Shibata, T. Sendo and Y. Gomita, Biol. Pharm. Bull., 2008, 31(2), 246-249.

16 Y. Kitamura, H. Araki and Y. Gomita, Pharmacol., Biochem. Behav., 2002, 71(1-2), 63-69.

17 K. Kawaura, Y. Ogata, S. Honda, F. Soeda, T. Shirasaki and K. Takahama, Behav. Brain Res., 2016, 302, 269-278.

18 K. Tokita, Y. Fujita, T. Yamaji and K. Hashimoto, Pharmacol., Biochem. Behav., 2012, 102(2), 329-334.

19 S. Bai, C. Zhou, P. Cheng, Y. Fu, L. Fang, W. Huang, J. Yu, W. Shao, X. Wang, M. Liu, J. Zhou and P. Xie, Int. J. Mol. Sci., 2015, 16(4), 8490-8504.

20 P. Zheng, Y. Wang, L. Chen, D. Yang, H. Meng, D. Zhou, J. Zhong, Y. Lei, N. D. Melgiri and P. Xie, Mol. Cell. Proteomics, 2013, 12(1), 207-214.

21 L. Liu, X. Zhou, Y. Zhang, Y. Liu, L. Yang, J. Pu, D. Zhu, C. Zhou and P. Xie, Behav. Brain Res., 2016, 305, 148-156.

22 G. Chen, D. Yang, Y. Yang, J. Li, K. Cheng, G. Tang, R. Zhang, J. Zhou, W. Li, Z. Liu, S. Fan and P. Xie, Behav. Brain Res., 2015, 278, 286-292.
23 X. Liu, P. Zheng, X. Zhao, Y. Zhang, C. Hu, J. Li, J. Zhao, J. Zhou, P. Xie and G. Xu, J. Proteome Res., 2015, 14(5), 2322-2330.

24 J. Chen, C. Zhou, P. Zheng, K. Cheng, H. Wang, J. Li, L. Zeng and P. Xie, Behav. Brain Res., 2017, 332, 280-287.

25 P. Zheng, Z. Fang, X. J. Xu, M. L Liu, X. Du, X. Zhang, H. Wang, J. Zhou and P. Xie, J. Affective Disord., 2016, 195, 75-81.

26 X. Zhou, L. Liu, Y. Zhang, J. Pu, L. Yang, C. Zhou, S. Yuan, H. Zhang and P. Xie, Neuroscience, 2017, 343, 1-9.

27 P. Willner, R. Muscat and M. Papp, Neurosci. Biobehav. Rev., 1992, 16(4), 525-534.

28 Q. Q. Mao, Z. Huang, S. P. Ip, Y. F. Xian and C. T. Che, Behav. Brain Res., 2012, 227(1), 305-309.

29 X. Wang, C. Zeng, J. Lin, T. Chen, T. Zhao, Z. Jia, X. Xie, Y. Qiu, M. Su, T. Jiang, M. Zhou, A. Zhao and W. Jia, J. Proteome Res., 2012, 11(12), 6223-6230.

30 X. Wang, T. Zhao, Y. Qiu, M. Su, T. Jiang, M. Zhou, A. Zhao and W. Jia, J. Proteome Res., 2009, 8(5), 2511-2518.

31 M. Li, X. Wang, J. Aa, W. Qin, W. Zha, Y. Ge, L. Liu, T. Zheng, B. Cao, J. Shi, C. Zhao, X. Wang, X. Yu, G. Wang and Z. Liu, Am. J. Physiol., 2013, 304(11), F1317-F1324.

32 Q. He, X. Kong, G. Wu, P. Ren, H. Tang, F. Hao, R. Huang, T. Li, B. Tan, P. Li, Z. Tang, Y. Yin and Y. Wu, Amino Acids, 2009, 37(1), 199-208.

33 Q. He, P. Ren, X. Kong, W. Xu, H. Tang, Y. Yin and Y. Wang, Mol. BioSyst., 2011, 7(7), 2147-2155.

34 Q. He, H. Tang, P. Ren, X. Kong, G. Wu, Y. Yin and Y. Wang, J. Proteome Res., 2011, 10(11), 5214-5221.

35 H. Blasco, J. Błaszczyński, J. C. Billaut, L. Nadal-Desbarats, P. F. Pradat, D. Devos, C. Moreau, C. R. Andres, P. Emond, P. Corcia and R. Słowiński, J. Biomed. Inf., 2015, 53, 291-299. 36 P. Willner, A. Towell, D. Sampson, S. Sophokleous and R. Muscat, Psychopharmacology, 1987, 93(3), 358-364.

37 S. Mahadevan, S. L. Shah, T. J. Marrie and C. M. Slupsky, Anal. Chem., 2008, 80(19), 7562-7570.

38 F. Zhang and G. Du, World J. Biol. Chem., 2012, 3(8), 167-174. 39 S. Huang, N. Chong, N. E. Lewis, W. Jia, G. Xie and L. X. Garmire, Genome Med., 2016, 8(2), 34.

40 X. Wang, A. Zhang, G. Yan, W. Sun, Y. Han and H. Sun, PLoS One, 2013, 8(8), e71403.

41 T. Iwai, T. Ohnuki, S. Sasaki-Hamada, A. Saitoh, A. Sugiyama and J. Oka, Behav. Brain Res., 2013, 243, 153-157.

42 B. Li, K. Suemaru, Y. Kitamura, Y. Gomita, H. Araki and R. Cui, J. Biochem. Mol. Toxicol., 2013, 27(11), 486-491.

43 J. Wu and Y. H. Gao, Expert Rev. Proteomics, 2015, 12(6), 623636.

44 M. A. Farag, N. M. Ammar, T. E. Kholeif, N. S. Metwally, N. M. El-Sheikh, L. A. Wessjohann and A. Z. Abdel-Hamid, Food Funct., 2017, 8(3), 985-996.

45 D. Yang, X. Wang, Y. Wu, B. Lu, A. Yuan, C. Leon and N. Guo, Molecules, 2015, 20(7), 11915-11929.

46 P. Elliott, J. M. Posma, Q. Chanx, I. Garcia-Perez, A. Wijeyesekera, M. Bictash, T. M. Ebbels, H. Ueshima, L. Zhao, L. van Horn, M. Daviglus, J. Stamler, E. Holmes and J. K. Nicholson, Sci. Transl. Med., 2015, 7(285), 285-262. 
47 A. Noto, G. Pomero, M. Mussap, L. Barberini, C. Fattuoni, F. Palmas, C. Dalmazzo, A. Delogu, A. Dessì, V. Fanos and P. Gancia, Ann. Transl. Med., 2016, 4(21), 417.

48 M. J. Owens and C. B. Nemeroff, Clin. Biochem., 1994, 40(2), 288-295.

49 R. P. Waters, M. Rivalan, D. A. Bangasser, J. M. Deussing, M. Ising, S. K. Wood, F. Holsboer and C. H. Summers, Neurosci. Biobehav. Rev., 2015, 58, 63-78.

50 S. Cabib and S. Puglisi-Allegra, Psychopharmacology1, 1996, 128(4), 331-342.

51 V. Krishnan and E. J. Nestler, Nature, 2008, 455(7215), 894902.

52 R. Odessey, E. A. Khairallah and A. L. Goldberg, J. Biol. Chem., 1974, 249(23), 7623-7629.

53 A. Zinellu, S. Sotgia, L. Deiana and C. Carru, Methods Mol. Biol., 2013, 919, 35-42.

54 J. S. Tian, G. J. Peng, Y. F. Wu, J. J. Zhou, H. Xiang, X. X. Gao, Y. Z. Zhou, X. M. Qin and G. H. Du, J. Chromatogr. B: Anal. Technol. Biomed. Life Sci., 2016, 1026, 227-235.

55 K. E. Tiedje, K. Stevens, S. Barnes and D. F. Weaver, Neurochem. Int., 2010, 57(3), 177-188.

56 J. Décombaz, M. Beaumont, J. Vuichoud, F. Bouisset and T. Stellingwerff, Amino Acids, 2012, 43(1), 67-76.

57 E. Martin, R. E. Rosenthal and G. Fiskum, J. Neurosci. Res., 2005, 79(1-2), 240-247.
58 B. Z. Li and Y. J. Yuan, Appl. Microbiol. Biotechnol., 2010, 86(6), 1915-1924.

59 S. J. Elferink, J. Krooneman, J. C. Gottschal, S. F. Spoelstra, F. Faber and F. Driehuis, Appl. Environ. Microbiol., 2001, 67(1), 125-132.

60 M. Hauptmann, D. F. Wilson and M. Erecinska, FEBS Lett., 1983, 161(2), 301-305.

61 J. S. Tian, B. Y. Shi, H. Xiang, S. Gao, X. M. Qin and G. H. Du, PLoS One, 2013, 8(9), e75721.

62 X. X. Gao, J. Cui, X. Y. Zheng, Z. Y. Li, Y. H. Choi, Y. Z. Zhou, J. S. Tian, J. Xing, X. J. Tan, G. H. Du and X. M. Qin, Phytother. Res., 2013, 27(7), 1074-1085.

63 U. E. Lang, C. Beglinger, N. Schweinfurth, M. Walter and S. Borgwardt, Cell. Physiol. Biochem., 2015, 37(3), 1029-1043.

64 J. Lu, Y. L. Zheng, D. M. Wu, L. Luo, D. X. Sun and Q. Shan, Biochem. Pharmacol., 2007, 74(7), 1078-1090.

65 A. Yildiz-Yesiloglu and D. P. Ankerst, Prog. NeuroPsychopharmacol. Biol. Psychiatry, 2006, 30(6), 969-995.

66 I. K. Yap, I. J. Brown, Q. Chan, A. Wijeyesekera, I. GarciaPerez, M. Bictash, R. L. Loo, M. Chadeau-Hyam, T. Ebbels, M. I. De, E. Maibaum, L. Zhao, H. Kesteloot, M. L. Daviglus, J. Stamler, J. K. Nicholson, P. Elliott and E. Holmes, J. Proteome Res., 2010, 9(12), 6647-6654. 